\title{
SHAPE VIA MULTI-NETS
}

\author{
By \\ Zvonko ČERIN
}

\begin{abstract}
We give in this paper a description of a new category related to shape category. We consider families of multi-valued functions between topological spaces which we call multi-nets. In a well-controlled way functions of a multi-net more and more resemble single-valued functions. We introduce a notion of homotopy for multi-nets and a composition of homotopy classes. The resultant homotopy category of multi-nets $\mathscr{H} M$ is naturally equivalent to the shape category provided we restrict to spaces which have ANRresolutions with onto projections. However, the homotopy category of multi-nets is interesting because it provides an intrinsic method of studying global properties of spaces. Our idea is to extend Borsuk's approach based on fundamental sequences to arbitrary topological spaces in analogy with Sanjurjo's description of shape category of compact metric spaces in terms of upper semi-continuous multi-valued functions.
\end{abstract}

\section{Introduction}

The subject of this paper belongs to the part of geometric topology which is known under the name shape theory. The method of our investigations is through the use of multi-valued functions. Our motivation is a desire to get a new description of the shape category which will be an extension to arbitrary topological spaces of Sanjurjo's approach to shape theory of compact metric spaces via upper semi-continuous multi-valued functions [10].

The classical homotopy theory studies the equivalence relation of homotopy for maps. Recall that maps (i. e., continuous single-valued functions) $f$ and $g$ between topological spaces $X$ and $Y$ are called homotopic provided there is a map $h$ from the product $X \times I$ of $X$ with the unit closed segment $I=[0,1]$ into

1980 Mathematics, Subject Classification (1985 Revision). Primary 54B25, 54F45, 54C56. Key words and phrases. homotopy, shape theory, multi-valued function, approximate resolution, expansion, $\sigma$-close, $\sigma$-small.

Received July 29, 1993. 
$Y$ such that $h(x, 0)=f(x)$ and $h(x, 1)=g(x)$ for every $x \in X$. The homotopy category $\mathscr{H}$ has as objects topological spaces and as morphisms homotopy classes of maps. The homotopy classes are composed by composing representatives and the identity morphisms are homotopy classes of the identity maps.

The equivalence relation of homotopy for maps leads to a useful and rich theory only when we restrict to spaces with nice local properties like polyhedra and absolute neighbourhood retracts. The problems arise in the definition above when the space $Y$ is such that there are not many maps from $X \times I$ into $Y$ so that the properties of $Y$ are preventing identification of maps which ought to be identified. In other words, the definition of homotopy is too rigid because the function $h$ must be continuous and single-valued and because it must take values in the space $Y$.

This has led K. Borsuk to modify homotopy theory so that the new theory which he calls shape theory agrees with the old on absolute neighbourhood retracts and that it gives much better results for spaces with complicated local structure where the old theory is inadequate. The modification of Borsuk relies on the idea to relinquish the insistence in the definition of homotopy that the map $h$ goes precisely into the space $Y$. The obvious alternative method which was undertaken by Sanjurjo in [9] and [10] and further followed in this paper is to give up with the requirement that the function $h$ is continuous and/or single-valued while retaining the desirable condition that it takes values in the space $Y$. In order to properly honour these two diverse methods we shall call them the Borsuk approach and the Sanjurjo approach to shape theory. We use names outer shape theory and inner shape theory.

In the original Borsuk's description [2] of shape category $S h_{B}$ of compact metric spaces, the spaces $X$ and $Y$ are considered as closed subsets of the Hilbert cube $Q$ and maps from $X$ into $Y$ are replaced with fundamental sequences. Recall that a fundamental seguence $\varphi$ from $X$ into $Y$ is a sequence $\left\{\varphi_{i}\right\}_{i=1}^{\infty}$ of maps $\varphi_{i}: Q \rightarrow Q$ such that for every neighbourhood $U$ of $Y$ in $Q$ there is a neighbourhood $V$ of $X$ in $Q$ and an index $i$ with the property that the restrictions $\left.\varphi_{i}\right|_{V}$ and $\left.\varphi_{j}\right|_{V}$ are homotopic in $U$ for every $j>i$. The role of the homotopy relation plays the following notion. Fundamental sequences $\varphi$ and $\phi$ from $X$ into $Y$ are called homotopic provided for every neighbourhood $U$ of $Y$ in $Q$ there is a neighbourhood $V$ of $X$ in $Q$ and an index $i$ with the property that the restrictions $\left.\varphi_{j}\right|_{V}$ and $\left.\phi_{j}\right|_{V}$ are homotopic in $U$ for every $j \geqq i$. This is an equivalence relation $[\varphi]$ denotes the homotopy class of a fundamental sequence $\varphi$, and homotopy classes are composed by the rule $[\psi] \circ[\varphi]=[\psi \circ \varphi]$, where $\phi \circ \varphi$ is a fundamental sequence formed by compositions $\psi_{i}{ }^{\circ} \varphi_{i}$. The category $S h_{B}$ has 
compact metric spaces as objects and homotopy classes of fundamental sequences as morphisms.

In spite of its simplicity and clear geometric flavour, Borsuk's theory relies too much on the Hilbert cube and the use of open neighbourhoods of subsets so that the extension of it to wider classes of spaces proved to be a formidable problem. This was accomplished by many authors so that now we have different descriptions of shape category $\mathcal{S}$. Its objects are topological spaces while its morphisms are rather awkward constructions involving things such as Morita's ANR-expansions, Grothendick's pro-categories, and intricate concepts of category theory (see [7]). All these efforts belong to the outer shape theory because they use some outside objects in order to study global properties of spaces. In particular, these approaches all require the use of absolute neighbourhood retracts. In this paper we propose to follow for arbitrary topological spaces Borsuk's geometric method based on fundamental sequences as closely as possible without any reference to absolute neighbourhood retracts.

Instead of fundamental sequences we consider multi-nets. The other steps are identical. We define a notion of homotopy for multi-nets and the morphisms are simply homotopy classes of multi-nets. This idea has previously been used by Sanjurjo in [9] and [10] to get an analogou description of $s h_{B}$. The crux of this approach is to use functions which are not continuous and/or singlevalued. Our investigation started with attempts to extend Sanjurjo's method to arbitray topological spaces.

The difference in approach is that we do not require multi-valued functions to be upper semi-continuous though it is possible with only minor modifications to build up the appropriate category where this requirement is fulfilled.

The key tools are given as Lemmas 2 and 3 which provide replacement of a small multi-valued functions (as defined in Definition 2) by a close (see Definition 3) continuous single-valued function and necessary transitivity of the notion of small homotopy (from Definition 4).

The multi-nets and their homotopy is given in Definitions 5 and 6 . The most difficult part is to find the correct notion of composition for homotopy classses of multi-nets. This is accomplished in the first three claims and summarized in Theorem 2.

With the description of the new category $\mathscr{H} M$ thus completed, the rest of the paper deals with setting up a functor $\theta$ from our homotopy category of multi-nets into the shape category (see Theorem 3 and Claims 4-6).

Finally, in Theorem 4 and Claims $7-10$, we show that the homotopy category of multi-nets is naturally equivalent to the shape category on spaces hav- 
ing ANR-resolutions with onto projections. This is done by describing the inverse $\zeta$ of the functor $\theta$.

The present paper is only the first in a series where we shall attempt to do large portions of inner shape theory using small multi-valued functions. This approach is particularly suitable for some problems. It's obvious merit is that it does not need any outside objects (like a nice ambient space or an inverse limit expansion into nice spaces). In conclusion, this paper lies foundations for the study of the homotopy category $\mathscr{H} M$ of multi-nets and establishes some connections between $\mathscr{H} M$ and the shape category. In the paper "Shape theory intrinsically" we shall prove by far more complicated arguments that the categories $\mathscr{H} M$ and $\mathcal{S h}$ are equivalent.

\section{Small Multi-valued functions}

In this section we shall introduce notions that are required for our theory and prove two useful technical results.

Let $\hat{Y}$ denote the collection of all normal covers of a topological space $Y$ [1]. With respect to the refinement relation $>$ the set $\hat{Y}$ is a directed set. Two normal covers $\sigma$ and $\tau$ of $Y$ are equivalent provided $\sigma>\tau$ and $\tau<\sigma$. In order to simplify our notation we denote a normal cover and it's equivalence class by the same symbol. Consequently, $\hat{Y}$ also stands for the associated quotient set.

Let $\hat{Y}$ denote the collection of all finite subsets $c$ of $\hat{Y}$ which have a unique (with respect to the refinement relation) maximal element $\tilde{c} \in \hat{Y}$. We consider $\tilde{Y}$ ordered by the inclusion relation and regard $\hat{Y}$ as a subset of single-element subsets of $\tilde{Y}$. Notice that $\tilde{Y}$ is a cofinite directed set $[7$, p. 11$]$.

We shall repeatedly use the following lemma (see $[7$, p. 9]).

LEMMA 1. Let $\left\{f_{1}, \cdots, f_{n}\right\}$ be a finite collection of functions from a cofinite directed set $(M,<)$ into a directed set $(L,<)$. Then there is an increasing function $g: M \rightarrow L$ such that $g(x)>f_{1}(x), \cdots, f_{n}(x)$ for every $x \in M$.

The next two definitions introduce precisely a type of multi-valued functions that we shall use.

Definition 1. Let $X$ and $Y$ be topological spaces. By a multi-valued function or an $M$-function $F: X \rightarrow Y$ we mean a rule which associates a non-empty subset $F(x)$ of $Y$ to every point $x$ of $X$. Let $M(X, Y)$ denote all $M$-functions from $X$ into $Y$. 
Definition 2. Let $F: X \rightarrow Y$ be a multi-valued function and let $\alpha \in \hat{X}$ and $\gamma \in \hat{Y}$. We shall say that $F$ is an $(\alpha, \gamma)$-map provided for every $A \in \alpha$ there is a $C_{A} \in \gamma$ with $F(A) \subset C_{A}$. On the other hand, $F$ is $\gamma$-small provided there is an $\alpha \in \hat{X}$ such that $F$ is an $(\alpha, \gamma)$-map.

The following is a notion of closeness for multi-valued functions that is needed in this approach to shape theory.

Definition 3. Let $F, G: X \rightarrow Y$ be multi-valued functions and let $\gamma \in \hat{Y}$. We shall say that $F$ and $G$ are $\gamma$-close and we write $F \stackrel{\gamma}{=} G$ provided for every $x \in X$ there is a $C_{x} \in \gamma$ with $F(x) \cup G(x) \subset C_{x}$.

The following lemma is not needed in the description of the category $\mathscr{H} M$ but only in setting up a functor $\theta$ from the category $\mathscr{H} M$ into the shape category $\mathcal{S}$. This is a very useful approximation result which shows that a sufficiently small multi-valued functions into an approximate polyhedron can be replaced by a continuous single-valued function.

Recall [7] that an approximate polyhedron is a topological space $Y$ with the property that for every $\sigma \in \hat{Y}$ there is a polyhedron $P$ and maps $u: Y \rightarrow P$ and $d: P \rightarrow Y$ with $i d_{Y} \stackrel{\sigma}{=} d \circ u$.

LEMMA 2. For every normal cover $\sigma$ of an approximate polyhedron $Y$ there is a normal cover $\tau$ of $Y$ such that every $\tau$-small multi-valued function $F: X \rightarrow Y$ from a topological space $X$ into $Y$ there is a normal cover $\rho$ of $X$ with the property that for every canonical map $p: X \rightarrow N(\rho)$ from $X$ into the nerve $N(\rho)$ of $\rho$ there is a single-valued continuous function $f: N(\rho) \rightarrow Y$ with $F \stackrel{o}{=} f \circ p$.

Proof of LemMa 2. Let $\lambda \in \sigma^{*}$ and $\nu \in \lambda^{*}$, where $\sigma^{*}$ denotes the set of all normal covers $\tau$ of $Y$ such that the star $\operatorname{st}(\tau)$ of $\tau$ refines $\sigma$. Choose a simplicial polytope $P$ with the metric topology and maps $u: Y \rightarrow P$ and $d: P \rightarrow Y$ with

$$
i d_{Y} \stackrel{\nu}{=} d \circ u \text {. }
$$

Let $\varepsilon=d^{-1}(\nu) \in \hat{P}$. Let $\eta \in \varepsilon^{*}$. Since $P$ is an ANR $[6$, p. 106], there is a refinement $\pi$ of $\eta$ with the property that every partial realization in $P$ of a simplicial polytope $K$ with the Whitehead topology relative to $\pi$ defined on a subpolytype $L$ of $K$ which contains all vertices of $K$ extends to a full realization of $K$ in $P$ relative to $\nu\left[6\right.$, p. 122]. Let $\xi \in \pi^{*}$ and let $\tau \in \hat{Y}$ be a common refinement of $\nu$ and $u^{-1}(\xi)$. 
Consider a $\tau$-small multi-valued function $F: X \rightarrow Y$. Choose a $\beta \in \hat{X}$ such that $F$ is a $(\beta, \tau)$-map. Let $\left\{\lambda_{B} \mid B \in \beta\right\}$ be a partition of unity subordinated to to $\beta$, and let $\left\{\mu_{B} \mid B \in \beta\right\}$ be its locally finite improvement $[4$, p. 354]. Let $\rho=$ $\left\{\mu_{B}^{-1}((0,1]) \mid B \in \beta\right\}$. Hence, for every $R \in \rho$ there is a $T_{R} \in \tau$, an $N_{R} \in \nu$, a $K_{R} \in \xi, y_{R} \in Y$ and a $z_{R} \in P$ with $F(R) \subset T_{R}, T_{R} \subset N_{R}, u\left(T_{R}\right) \subset K_{R}, y_{R} \in T_{R}, z_{R} \in K_{R}$ and $z_{R}=u\left(y_{R}\right)$. Let $p: X \rightarrow N(\rho)$ be a canonical map of $X$ into the nerve $N(\rho)$ of $\rho$ (see [4]).

Define a function $\varphi: N(\rho)^{0} \rightarrow P$ by the rule $\varphi(R)=z_{R}$ for every $R \in \rho$. This function is continuous and it provides a partial realization of $N(\rho)$ in $P$ relative to the cover $\pi$.

Indeed, let $\delta=\langle A, B, \cdots, Z\rangle$ be a simplex of $N(\rho)$. We shall find a member of $\pi$ which contains the set $\varphi\left(N(\rho)^{0} \cap \delta\right)$, i. e., the set $\left\{z_{A}, \cdots, z_{Z}\right\}$. Suppose $x \in A \cap \cdots \cap Z$. Since $F(x)$ is non-empty, the sets $T_{A}, \cdots, T_{z}$ and therefore also the sets $K_{A}, \cdots, K_{Z}$ have non-empty intersection. Since $\xi$ is a star-refinement of $\pi$, it is clear that some member $P_{\delta}$ of $\pi$ contains their union.

Let $\phi: N(\rho) \rightarrow Y$ be a full realization in $P$ of $N(\rho)$ relative to $\eta$. Let $f$ denote the composition $d \circ \phi$. Then $f$ is the required continuous single-valued function.

Let $x \in X$ and suppose that $A, \cdots, Z$ are all members of $\rho$ which contain the point $x$. Then $p(x)$ lies in the simplex $\delta$ of $N(\rho)$ determined by these sets. It follows that a member $E_{x}$ of $\eta$ contains both $\phi \circ p(x)$ and points $z_{A}, \cdots, z_{z}$. Since $\xi$ refines $\eta$ and $\eta$ is a star-refinement of $\varepsilon$, there is a member $N_{x}$ of $\nu$ with $d\left(E_{x} \cup K_{A} \cup \cdots \cup K_{Z}\right) \subset N_{x}$. On the other hand, from (1) we get the existence of members $N_{A}, \cdots, N_{Z}$ of $\nu$ such that $N_{C}$ contains both $y_{C}$ and $d\left(z_{C}\right)$ for every $C=A, \cdots, Z$. It follows that

$$
f \circ p(x) \in N_{x} \quad d\left(z_{A}\right) \in N_{x} \cap N_{A}, \quad y_{A} \in N_{A} \cap T_{A}, \quad F(x) \subset T_{A} .
$$

Hence, some member of $\sigma$ contains both $f \circ p(x)$ and $F(x)$.

The following definition is the most important for this paper and our approach to inner shape theory.

Definition 4. Let $F, G: X \rightarrow Y$ be multi-valued functions between topological spaces and let $\gamma$ be a normal cover of the space $Y$. We shall say that $F$ and $G$ are $\gamma$-homotopic and write $F \stackrel{\gamma}{\cong} G$ provided there is a $\gamma$-small multivalued function $H$ from the product $X \times I$ of $X$ and the unit segment $I=[0,1]$ into $Y$ such that $F(x) \subset H(x, 0)$ and $G(x) \subset H(x, 1)$ for every $x \in X$. We shall say that $H$ is a $\gamma$-homotopy that joins $F$ and $G$ or that it realizes the relation 
(or homotopy) $F \stackrel{r}{\cong} G$.

The following lemma gives an adequate substitute for transitivity of the homotopy relation for maps. It will be used later many times.

LeMmA 3. Let $F, G, H: X \rightarrow Y$ be multi-valued functions. Let $\sigma \in \hat{Y}$ and $\tau \in \sigma^{*} . \quad$ If $F \stackrel{\tau}{\cong} G$ and $G \stackrel{\tau}{\cong} H$, then $F \stackrel{\sigma}{\cong} H$.

Proof of Lemma 3. Let $K, L: X \times I \rightarrow Y$ be $\tau$-small multi-valued functions such that

$$
F(x) \subset K(x, 0), \quad G(x) \subset K(x, 1) \cap L(x, 0), \quad H(x) \subset L(x, 1)
$$

for every $x \in X$. Define $M: X \times I \rightarrow Y$ by

$$
M(x, t)= \begin{cases}K(x, 2 t), & x \in X, 0 \leqq t<1 / 2 \\ K(x, 1) \cup L(x, 0), & x \in X, t=1 / 2 \\ L(x, 2 t-1), & x \in X, 1 / 2<t \leqq 1 .\end{cases}
$$

Clearly, by (2), $F(x) \subset M(x, 0)$ and $H(x) \subset M(x, 1)$ for every $x \in X$. Hence, it remains to see that $M$ is $\sigma$-small.

Since both $K$ and $L$ are $\tau$-small, there are normal covers $\alpha$ and $\beta$ of $X \times I$ so that for every $A \in \alpha$ there is a $T_{A}^{K} \in \tau$ with $K(A) \subset T_{A}^{K}$ and for every $B \in \beta$ there is a $T_{B}^{L} \in \tau$ with $L(B) \subset T_{B}^{L}$. Let a normal cover $\gamma$ be a common refinement of $\alpha$ and $\beta$. Then for every $C \in \gamma$ we can find $T(C), W(C) \in \tau$ with

$$
K(C) \subset T(C) \text { and } L(C) \subset W(C) \text {. }
$$

We now use $[4$, p. 358], to get a normal cover $\varepsilon \in \hat{X}$ together with the function $r: \varepsilon \rightarrow\{2,3,4, \cdots\}$ such that every set $E \times\left[t_{2 i}, t_{2 i+4}\right]$ is contained in a member $C_{E, i}$ of $\gamma$, where $E \in \varepsilon, i=0,1,2, \cdots, r E-2$, and $t_{j}=j / 4 r E$ for every $j=0,1, \cdots, 4 r E$.

We define for each $E \in \varepsilon$ an open over $|E|$ of $I$ as follows:

$$
|E|=\left\{V_{1}, V_{2}, \cdots, V_{4 r E-1}\right\},
$$

where $V_{1}=\left[0, t_{2}\right), V_{2}=\left(t_{1}, t_{3}\right) V_{3}=\left(t_{2}, t_{4}\right), \cdots, V_{4 r E-1}=\left(t_{4 r E-2}, 1\right]$.

Since $\{\{E \times V|V \in| E \mid\} \mid E \in \varepsilon\}$ is a normal cover of $X \times I$, our proof will be completed provided we show that for every $E \in \varepsilon$ and every $V \in|E|$ there is a member of $\sigma$ containing $M(E \times V)$.

If $V=V_{i}$, for $i \neq 2 r E$, this follows from (3).

Let $V=V_{2 r E}$. Then $M(E \times V)=K\left(E \times\left(t_{4 r E-2}, 1\right]\right) \cup L\left(E \times\left[0, t_{2}\right)\right)$. But, $K(E \times$ $\left.\left(t_{4 r E-2}, 1\right]\right) \subset T\left(C_{E, 2 r E-1}\right)$ and $L\left(E \times\left[0, t_{2}\right)\right) \subset W\left(C_{E, 0}\right)$. As $E$ is a non-empty set, 
there is an $x \in E$. The relation (2) shows that the non-empty set $G(x)$ lies in the intersection of sets $T\left(C_{E, 2 r E-1}\right)$ and $W\left(C_{E, 0}\right)$. Hence. a member of $\sigma$ contains $M(E \times V)$.

\section{Multi-nets}

The following two definitions correspond to Borsuk's definitions of fundamental sequences and homotopy for fundamental sequences.

Definition 5. Let $X$ and $Y$ be topological spaces. By a multi-net or an $M$-net from $X$ into $Y$ we shall mean a collection $\varphi=\left\{F_{c} \mid c \in \tilde{Y}\right\}$ of multi-valued functions $F_{c}: X \rightarrow Y$ such that for every $\gamma \in \hat{Y}$ there is a $c \in \tilde{Y}$ with $F_{d} \stackrel{\Upsilon}{\cong} F_{c}$ for every $d>c$. We use functional notation $\varphi: X \rightarrow Y$ to indicate that $\varphi$ is a multinet from $X$ into $Y$. Let $M N(X, Y)$ denote all multi-nets $\varphi: X \rightarrow Y$.

Definition 6. Two multi-nets $\varphi=\left\{F_{c}\right\}$ and $\phi=\left\{G_{c}\right\}$ between topological spaces $X$ and $Y$ are homotopic provided for every $\gamma \in \hat{Y}$ there is a $c \in \tilde{Y}$ such that $F_{d} \stackrel{\gamma}{\cong} G_{d}$ for every $d>c$.

It follows from Lemma 3 that the relation of homotopy is an equivalence relation on the set $M N(X, Y)$. The homotopy class of a multi-net $\varphi$ is denoted by $[\varphi]$ and the set of all homotopy classes by $\mathscr{H} M(X, Y)$.

Our first goal is to define a composition for homotopy classes of multi-nets and to establish its associativity.

Let $\varphi=\left\{F_{c}\right\}: X \rightarrow Y$ be a multi-net. For every $c \in \tilde{Y}$ there is an $\bar{f}(c) \in \tilde{Y}$ such that for all $d, e>\bar{f}(c)$ there is a normal cover $\bar{f}(c, d, e)$ of $X \times I$ and an $(\bar{f}(c, d, e), \tilde{c})$-map joining $F_{d}$ and $F_{e}$.

Let $\mathcal{C}=\{(c, d, e) \mid c \in \tilde{Y}, d, e>\bar{f}(c)\}$. Then $\mathcal{C}$ is a subset of $\tilde{Y} \times \tilde{Y} \times \tilde{Y}$ that becomes a cofinite directed set when we define that $(c, d, e)>\left(c^{\prime}, d^{\prime}, e^{\prime}\right)$ iff $c>c^{\prime}, d>d^{\prime}$ and $e>e^{\prime}$.

Now, let $f: \tilde{Y} \rightarrow \tilde{Y}$ be an increasing function such that $f(c)>\bar{f}(c), c$ for every $c \in \tilde{Y}$. We shall use the same notation $f$ for an increasing function $f: \mathcal{C} \rightarrow \widehat{X \times I}$ such that $f(c, d, e)>\bar{f}(c, d, e)$ for every $(c, d, e) \in \mathcal{C}$. Let $(c, d, e)$ $\in \mathcal{C}$. For the normal cover $f(c, d, e)$ of $X \times I$, by [4, p. 358], there is a normal cover $\varepsilon=\hat{f}(c, d, e)$ of $X$ and a function $r=\tilde{f}(c, d, e): \varepsilon \rightarrow\{2,3,4, \cdots\}$ such that every set $E \times[(i-1) / r E,(i+1) / r E]$, where $E \in \varepsilon$ and $i=1,2, \cdots, r E-1$, is contained in a member of $f(c, d, e)$.

Let $\tilde{f}: \mathcal{C} \rightarrow \hat{X}$ be an increasing function with $\tilde{f}(c, d, e)>\hat{f}(c, d, e)$ for every 
$(c, d, e) \in \mathcal{C}$. We shall use the shorter notation $\tilde{f}(c)$ and $f(c)$ for the covers $\tilde{f}(c, f(c), f(c))$ and $f(c, f(c), f(c))$.

Claim 1. There is an increasing function $f^{*}: \tilde{Y} \rightarrow \hat{X}$ such that

(1) $f^{*}(c)>\tilde{f}(c)$ for every $c \in \tilde{Y}$, and

(2) $f^{*}$ is cofinal in $\tilde{f}$, i.e., for every $(c, d, e) \in \mathcal{C}$ there is an $m \in \tilde{Y}$ with $f^{*}(m)>\tilde{f}(c, d, e)$.

Proof of Claim 1. Let $\mathscr{D}=\{\tilde{f}(c, d, e) \mid(c, d, e) \in \mathcal{C}\}$.

If $\tilde{Y}$ is a finite set, then $\mathscr{D}$ is a finite collection of elements of $\hat{X}$. Let $\alpha \in \hat{X}$ be a common refinement of all members of $\mathscr{D}$. Let $f^{*}: \tilde{Y} \rightarrow \hat{X}$ be a constant function into $\alpha$.

If $\tilde{Y}$ is an infinite set, then the cardinality of $\mathscr{D}$ does not exceed the cardinality of $\tilde{Y}$. Hence, there is a surjection $g: \tilde{Y} \rightarrow \mathscr{D}$. Let $f^{*}: \tilde{Y} \rightarrow \hat{X}$ be an increasing function such that $f^{*}(c)>g(c), \tilde{f}(c)$ for every $c \in \tilde{Y}$.

The above discussion shows that every multi-net $\varphi: X \rightarrow Y$ determines eight functions denoted by $\bar{f}, f, \hat{f}, \tilde{f}$ and $f^{*}$. With the help of these functions we shall define the composition of homotopy classes of multi-nets as follows.

Let $\varphi=\left\{F_{c}\right\}: X \rightarrow Y$ and $\phi=\left\{G_{s}\right\}: Y \rightarrow Z$ be multi-nets. Let $\chi=\left\{H_{s}\right\}$, where $H_{s}=G_{g(s)} \circ F_{f(g *(s))}$ for every $s \in \tilde{Z}$.

Claim 2. The collection $\chi$ is a multi-net from $\boldsymbol{X}$ into $Z$.

Proof of Claim 2. Let $\sigma \in \hat{Z}$. We must find an $s \in \tilde{Z}$ such that

$$
H_{t} \stackrel{\sigma}{\cong} H_{s} \quad \text { for every } t>s .
$$

Let $\tau \in \sigma^{* 2}$, where $\sigma^{* n}$ denotes the set of all normal covers $\tau$ of $Z$ such that the $n$-th $\operatorname{star} s t^{n}(\tau)$ of $\tau$ refines $\sigma$. Let $s=\{\tau\} \in \tilde{Z}$.

Consider an index $t>s$. We shall find an index $c \in \tilde{Y}$ so that

$$
\begin{gathered}
H_{t} \stackrel{\tau}{\cong} G_{x} \circ F_{c}, \\
G_{x} \circ F_{c} \stackrel{\tau}{\cong} G_{y} \circ F_{c},
\end{gathered}
$$

and

$$
G_{y} \circ F_{c} \stackrel{\tau}{\cong} H_{s},
$$

where $x=g(t)$ and $y=g(s)$. Repeated use of Lemma 3 will give (13) from the relations $(14)-(16)$. 
Invoking the property (2) of Claim 1 , choose a $u>t$ so that $r>\tilde{g}(s, p, q)$, where $r=g^{*}(u), p=g(s)$, and $q=g(t)$. Let $c=f(r)$. Since $q>p>\bar{g}(s)$ and $\tilde{s}=\tau$, there is a $(g(s, p, q), \tau)$-map $L: Y \times I \rightarrow Z$ joining $G_{p}$ and $G_{q}$. But, $F_{c}$ is joined to itself by an $r$-small homotopy. It follows that $L \circ\left(F_{c} \times i d_{I}\right)$ is a $\tau$-small homotopy realizing the relation (15).

On the other hand, $G_{x}$ is a $(\tilde{g}(t), \tau)$-map while $F_{f\left(g^{*}(t)\right)}$ and $F_{c}$ are joined by a $g^{*}(t)$-small homotopy $K$. The property (1) of Claim 1 implies that $G_{g(t)} \circ K$ is a $\tau$-small homotopy which realizes the relation (14). In an analogous fashion one can show that (16) is also true.

We now define the composition of homotopy classes of multi-nets by the rule $\left[\left\{G_{s}\right\}\right] \circ\left[\left\{F_{c}\right\}\right]=\left[\left\{G_{g(s)} \circ F_{f(g *(s))}\right\}\right]$.

CLAIM 3. The composition of homotopy classes of multi-nets is well-defined.

Proof of Claim 3. Let $\kappa=\left\{K_{c}\right\}$ and $\lambda=\left\{L_{s}\right\}$ be multi-nets homotopic to $\varphi$ and $\phi$, respectively, and let $\mu=\left\{M_{s}\right\}$, where $M_{s}=L_{l(s)} \circ K_{k(l *(s))}$ for every $s \in \tilde{Z}$. We must show that multi-nets $\chi$ and $\mu$ are homotopic. In other words, that for every $\sigma \in \hat{Z}$ there is an $s \in \tilde{Z}$ such that

$$
H_{t} \stackrel{\tau}{\cong} M_{t} \quad \text { for every } t>s .
$$

Let $\sigma \in \hat{Z}$. Let $\tau \in \sigma^{* 4}$. Let $s=\{\tau\} \in \tilde{Z}$. In order to prove (17), we shall argue that for every $t>s$ we can find indices $c \in \tilde{Y}$ and $u \in \tilde{Z}$ such that

$$
\begin{aligned}
& H_{t} \stackrel{\tau}{\cong} G_{x} \circ F_{c}, \\
& G_{x} \circ F_{c} \stackrel{\tau}{\cong} G_{u} \circ F_{c}, \\
& G_{u} \circ F_{c} \stackrel{\tau}{\cong} L_{u} \circ F_{c}, \\
& L_{u} \circ F_{c} \stackrel{\tau}{\cong} L_{u} \circ K_{c}, \\
& L_{u} \circ K_{c} \stackrel{\tau}{\cong} L_{y} \circ K_{c}, \\
& L_{y} \circ K_{c} \stackrel{\tau}{\cong} M_{t},
\end{aligned}
$$

where we put $x=g(t)$ and $y=l(t)$. From the relations (18)-(23) with the help of Lemma 3 we shall get (17).

We shall now describe how big $c$ and $u$ must be chosen for relations (18), (19), (20) and (21) to hold separately. The relations (22) and (23) are analogous to relations (19) and (18), respectively. We leave to the reader the task of making a cumulative choice for $c$ and $u$ which accomplishes our goal. It is 
important to notice that $u$ is selected first while $c$ is selected only once $u$ is already known.

Add (18). Since $G_{x}$ is a $(\tilde{g}(t), \tau)$-map and $g *(t)$ refines the cover $\tilde{g}(t)$, by the property (1) of Claim 1 , it sufflces to take $c>f\left(g^{*}(t)\right)$.

Add (19). If $u>x$, then $G_{x}$ and $G_{u}$ are joined by a $(g(u), \tau)$-map $P: Y \times$ $I \rightarrow Z$. Let $c>f\left(g^{*}(t)\right)$. Then $F_{c}$ is $g^{*}(u)$-small. Since $g^{*}(u)>\tilde{g}(u)$, it follows that $P \circ\left(F_{c} \times i d_{I}\right)$ is a $\tau$-small homotopy joining the left and the right side of the relation (19).

Add (20). Since $\phi \cong \lambda$, there is a $u \in \tilde{Z}$, a normal cover $\eta$ of $Y \times I$, and an $(\eta, \tau)$-map $S: Y \times I \rightarrow Z$ joining $G_{u}$ and $L_{u}$. Let $\xi$ be a normal cover of $Y$ obtained by the application of [4, p. 358] to the cover $\eta$. If $c>f(\xi)$, then $F_{a}$ is $\xi$-small so that $S \circ\left(F_{c} \times i d_{I}\right)$ is a $\tau$-small homotopy joining compositions which appear in (20).

Add (21). Let $u>y$. Then $L_{u}$ is an $(\tilde{l}(u), \tau)$-map. Since $\varphi$ and $\kappa$ are homotopic, there is an index $c \in C$ so that $F_{c}$ and $K_{c}$ are joined by an $\tilde{l}(u)$ small homotopy $T: X \times I \rightarrow Y$. The composition $L_{u} \circ T$ realizes the relation (21).

THEOREM 1. The composition of homotopy classes of multi-nets is associative.

Proof of TheOREM 1. Let $\varphi=\left\{F_{c}\right\}, \phi=\left\{G_{s}\right\}$ and $\chi=\left\{H_{p}\right\}$ be multi-nets from $X$ into $Y$, from $Y$ into $Z$, and from $Z$ into $W$, respectively. Let $\mu=\left\{M_{s}\right\}$, $\nu=\left\{N_{p}\right\}, \kappa=\left\{K_{p}\right\}$ and $\lambda=\left\{L_{p}\right\}$, where $M_{s}=G_{g(s)} \circ F_{f(g *(s))}$ for every $s \in \tilde{Z}$ and $N_{p}=H_{h(p)} \circ G_{g(h *(p)),} K_{p}=H_{h(p)} \circ M_{m(h *(p))}$, and $L_{p}=N_{n(p)} \circ F_{f(n *(p))}$, for every $p \in \widetilde{W}$. We must show that $\kappa$ and $\lambda$ are homotopic, i. e., that for every $\pi \in \hat{W}$ there is a $p \in \widetilde{W}$ such that

$$
K_{q} \stackrel{\pi}{\cong} L_{q} \quad \text { for every } q>p
$$

Let $\pi \in \hat{W}$. Let $\rho \in \pi^{* 4}$. Let $p=\{\rho\} \in W$. In order to prove (24), we shall show that for every $q>p$ we can find indices $c \in \tilde{Y}$ and $s \in \tilde{Z}$ such that

$$
\begin{gathered}
K_{q} \stackrel{\rho}{\cong} H_{x} \circ G_{y} \circ F_{c}, \\
H_{x} \circ G_{y} \circ F_{c} \stackrel{\rho}{\cong} H_{x} \circ G_{s} \circ F_{c}, \\
H_{x} \circ G_{s} \circ F_{c} \stackrel{\rho}{\cong} H_{z} \circ G_{s} \circ F_{c}, \\
H_{z} \circ G_{s} \circ F_{c} \stackrel{\rho}{\cong} N_{w} \circ F_{c},
\end{gathered}
$$

and 


$$
N_{w} \circ F_{c} \stackrel{\rho}{\cong} L_{q},
$$

where $x=h(q), y=g(m(h *(q))), z=h(n(q))$ and $w=n(q)$. Repeated use of Lemma 3 will give (24) from the relations (25)-(29).

The method of proof is similar to the proof of Claim 3. We shall only describe for each of the relations (25)-(29) how large the indices $u$ and $c$ must be in order that this homotopy holds. An easy exercise of putting together all these selections is once again left to the reader. Since relations (28) and (29) are analogous with relations (26) and (25), respectively, it suffices to consider only relations (25)-(27).

Add (25). Observe that $H_{x}$ is an $(\alpha, \rho)$-map while $G_{y}$ is a $(\beta, \alpha)$-map, where $\alpha=\tilde{h}(q), \beta=h^{*}(q)$, and $\gamma=\tilde{g}(m(\beta))$. Let $\delta=g^{*}(m(\beta))$. If $c>f(\delta)$, then $F_{f(\delta)}$ and $F_{c}$ are joined by a $\delta$-small homotopy $P: X \times I \rightarrow Y$. But, $\delta$ refines $\gamma$ by the property (1) of Claim 1. Hence, $H_{x} \circ G_{y} \circ P$ is a $\rho$-small homotopy between $K_{q}$ and $H_{x} \circ G_{y} \circ F_{c}$.

Add (26). As above, $H_{x}$ is an $(\alpha, \rho)$-map. Since $m(s)>s$ for every $s \in \tilde{Z}$, we get $y>g(\beta)$. Therefore, if we take $s>y$, then $G_{y}$ and $G_{s}$ are joined by an $(\varepsilon, \beta)$-map $Q: Y \times I \rightarrow Z$, where $\varepsilon=g(\beta)$. However, $\beta$ refines $\alpha$ so that $H_{x} \circ Q$ is an $(\varepsilon, \rho)$-map. Let $\eta$ be a normal cover of $Y$ associated to $\varepsilon$ by $[4$, p. 358]. Finally, for $c>f(\eta)$ we see that $H_{x} \circ Q \circ\left(F_{c} \times i d_{I}\right)$ realizes the relation (26).

Add (27). Since $n(r)>r$ for every $r \in \widetilde{W}$, we get $z>x$ so that $H_{x}$ and $H_{z}$ are joined by an $(\eta, \rho)$-map $T: Z \times I \rightarrow W$, where $\eta$ denotes the normal cover $h(z)$ of $Z \times I$. Let $\xi=h *(z)$ and let $s>g(\xi)$. Then $G_{s}$ is a $(\tilde{g}(s), \xi)$-map. Let $\zeta=g^{*}(s)$ and take $c>f(\zeta)$. The composition $T \circ\left(\left(G_{s} \circ F_{c}\right) \times i d_{I}\right)$ realizes the relation (27).

\section{The category $\mathscr{H} M$}

For a topological space $X$, let $c^{X}=\left\{I_{a}\right\}: X \rightarrow X$ be the identity multi-net defined by $I_{a}=i d_{X}$ for every $a \in \tilde{X}$. It is easy to show that for every multinet $\varphi: X \rightarrow Y$ the following relations hold.

$$
[\varphi] \circ\left[\iota^{X}\right]=[\varphi]=\left[\iota^{Y}\right] \circ[\varphi]
$$

We can summarize the above with the following theorem.

THEOREM 2. The topological spaces as objects together with the homotopy classes of multi-nets as morphisms and the composition of homotopy classes form the category $\mathscr{H} M$. 
There is an obvious functor $J$ from the category Iop of topological spaces and continuous maps into the category $\mathscr{H} M$. On objects the functor $J$ is the identity while on morphisms it associates to a map $f: X \rightarrow Y$ the homotopy class of a multi-net $\underline{f}=\left\{F_{c}\right\}: X \rightarrow Y$, where $F_{c}=f$ for every $c \in \tilde{Y}$.

Our first main result can be stated as follows. Let $S h$ be the shape category of arbitrary topological spaces and let $S: \mathscr{I} o p \rightarrow \mathcal{S h}$ be the shape functor [7].

THEOREM 3. There is a functor $\theta$ from the category $\mathscr{H} M$ into the shape category sh such that $S=\theta \circ J$.

\section{Description of the functor $\theta$}

The functor $\theta$ will leave the objects unchanged. In order to explain how $\theta$ effects the morphisms we must work much harder. First we encounter the dilemma of selecting the right description of shape morphisms among the many that exist in the literature.

In the rest of this paper, let $X, Y$ and $Z$ be topological spaces and let

and

$$
\begin{aligned}
& \boldsymbol{p}=\left\{p^{a}\right\}: X \longrightarrow \mathscr{X}=\left\{X_{a}, \boldsymbol{\varepsilon}_{a}, p_{b}^{a}, A\right\}, \\
& \boldsymbol{q}=\left\{q^{c}\right\}: Y \longrightarrow \mathcal{Y}=\left\{Y_{c}, \boldsymbol{\xi}_{c}, q_{d}^{c}, C\right\},
\end{aligned}
$$

$$
\boldsymbol{r}=\left\{r^{m}\right\}: Z \longrightarrow \mathscr{Z}=\left\{Z_{m}, \nu_{m}, r_{n}^{m}, M\right\}
$$

be uniform commutative approximate resolutions of $X, Y$ and $Z$ where each $X_{a}, Y_{c}$ and $Z_{m}$ is a polyhedron, $s t^{3}\left(\varepsilon_{a}\right)$-close maps into $X_{a}, s t^{3}\left(\xi_{c}\right)$-close maps into $Y_{c}$ and $s t^{3}\left(\nu_{m}\right)$-close maps into $Z_{m}$ are homotopic, and $A=(A,>), C=(C,>)$, and $M=(M,>)$ are infinite cofinite directed sets with cardinalities greater or equal to cardinalities of $\tilde{X}, \tilde{Y}$ and $\tilde{Z}$, respectively. The existence of such approximate resolutions follows from [8] and [11].

We can associate with the approximate resolutions $\boldsymbol{p}, \boldsymbol{q}$ and $\boldsymbol{r}$ the underlying expansions in the sense of Morita [7]

and

$$
\begin{aligned}
& |\boldsymbol{p}|=\left\{p^{a}\right\}: X \longrightarrow|\mathscr{X}|=\left\{X_{a}, p_{b}^{a}, A\right\}, \\
& |\boldsymbol{q}|=\left\{q^{c}\right\}: Y \longrightarrow\left|g_{a}\right|=\left\{Y_{c}, q_{a}^{c}, C\right\},
\end{aligned}
$$

$$
|\boldsymbol{r}|=\left\{r^{m}\right\}: Z \longrightarrow|\mathcal{Z}|=\left\{Z_{m}, r_{n}^{m}, M\right\} .
$$

It is well-known that shape morphisms from $X$ into $Y$ could be considered as equivalence classes of morphisms of inverse systems $|\mathscr{X}|$ and $|\mathscr{y}|$ (see [7] and [11]). More precisely, the set $\operatorname{sh}(X, Y)$ of all shape morphisms between 
spaces $X$ and $Y$ can be identified with the set pro- $\mathscr{P} \operatorname{Pol}(|\mathfrak{X}|,|\mathscr{y}|)$ of all morphisms in the Grothendick's pro-category pro- $\mathscr{H} P o l$ of the homotopy of polyhedra $\mathscr{H} P o l$ between the objects $|\mathscr{X}|$ and $|\mathscr{Y}|$. In our description of what $\theta$ does on morphisms of the category $\mathscr{H} M$ we shall view shape morphisms in this way.

Let $\varphi=\left\{F_{s}\right\}_{s \in \bar{Y}}: X \rightarrow Y$ be a multi-net. By Lemma 2, we can find a refinement $\eta_{c}$ of $\xi_{c}$ so that for every $s t^{2}\left(\eta_{c}\right)$-small multi-valued function $K: W \rightarrow Y_{c}$ there is a normal cover $\rho$ of $W$ with the property that for every canonical map $r: W \rightarrow N(\boldsymbol{\rho})$ there is a map $k: N(\boldsymbol{\rho}) \rightarrow Y_{c}$ with $K \stackrel{\xi_{c}}{=} k \circ r$. Let $\pi_{c}=\left(q^{c}\right)^{-1}\left(\eta_{c}\right)$.

Choose an index $l_{c} \in \tilde{Y}$ so that

$$
F_{s} \stackrel{\pi_{c}}{\cong} F_{t} \quad \text { for all } s, t>l_{c}
$$

Let $\lambda: C \rightarrow \tilde{Y}$ be an increasing function such that $\lambda(c)>l_{c},\left\{\pi_{c}\right\}, v(c)$ for every $c \in C$, where $v: C \rightarrow \tilde{Y}$ is a surjection. We shall need later the fact that the function $\lambda$ is cofinal, i.e., that for every $s \in \tilde{Y}$ there is a $d \in C$ with $\lambda(d)>s$.

Observe that $F_{\lambda(c)}$ is $\pi_{c}$-small. Hence, $q^{c} \circ F_{\lambda(c)}$ is $\eta_{c}$-small. Let $\rho$ be a normal cover of $X$ such that $q^{c} \circ F_{\lambda(c)}$ is a $\left(\rho, \eta_{c}\right)$-map. Let $r: X \rightarrow N(\rho)$ be a canonical map. The way in which we selected the cover $\eta_{c}$ gives a map $k$ which satisfies

$$
k \circ r \stackrel{\xi_{c}}{=} q^{c} \circ F_{\lambda(c)}
$$

Let $\zeta=k^{-1}\left(\boldsymbol{\xi}_{c}\right)$. By the property (R1) for the approximate resolution $\boldsymbol{p}$, there is an index $f(c) \in A$ and a map $g: X_{f(c)} \rightarrow N(\rho)$ with $r \stackrel{\zeta}{=} g \circ p^{f(c)}$. Hence,

$$
k \circ r \stackrel{\xi_{c}}{=} k \circ g \circ p^{f(c)} \text {. }
$$

Let $f^{c}=k \circ g: X_{f(c)} \rightarrow Y_{c}$. The relations (32) and (33) together imply

$$
f^{c} \circ p^{f(c)} \stackrel{s t\left(\xi_{c}\right)}{=} q^{c} \circ F_{\lambda(c)} .
$$

ClaIM 4. The pair $\boldsymbol{f}=\left(f,\left\{f^{c} \mid c \in C\right\}\right)$ is a morphism between inverse systems $|\mathfrak{X}|$ and $|a|$ |

Proof of Claim 4. We must show that for every pair $c, d$ of elements of $C$ with $d>c$ it is passible to find an $a>f(c), f(d)$ so that

$$
f^{c} \circ p_{a}^{f(c)} \cong q_{d}^{c} \circ f^{d} \circ p_{a}^{f(d)} .
$$

Since $\lambda(d)>\lambda(c)>l_{c}$, by (31), the functions $F_{\lambda(c)}$ and $F_{\lambda(d)}$ can be joined by $\pi_{c^{-}}$ small homotopy $H: X \times I \rightarrow Y$. Hence, $q^{c} \circ F_{\lambda(c)}$ and $q^{c} \circ F_{\lambda(d)}$ are joined by the $\eta_{c^{-}}$ 
small homotopy $q^{c} \circ H$. It follows that there is a single-valued continuous function $K: X \times I \rightarrow Y$ with

$$
K \stackrel{\xi_{c}}{=} q^{c} \circ H
$$

The way in which we constructed $f^{d}$, the relation $q^{c}=q_{d}^{c} \circ q^{d}$, and the uniformity property of $\boldsymbol{q}$ give

$$
q^{c} \circ F_{\lambda(d)} \stackrel{s t\left(\xi_{c}\right)}{=} q_{d}^{c} \circ f^{d} \circ p^{f(d)}
$$

We know that

$$
q^{c} \circ F_{\lambda(c)}(x) \subset q^{c} \circ H(x, 0) \text { and } q^{c} \circ F_{\lambda(d)}(x) \subset q^{c} \circ H(x, 1)
$$

for every $x \in X$. Combining relations (36), (34) and (38) we obtain

$$
K_{0} \stackrel{s t^{2}\left(\xi_{c}\right)}{=} f^{c} \circ p^{f(c)}
$$

while (36), (37) and (38) imply

$$
K_{1} \stackrel{s t^{2}\left(\xi_{c}\right)}{=} q_{d}^{c} \circ f^{d} \circ p^{f(d)},
$$

where $K_{0}, K_{1}: X \rightarrow Y$ are defined by $K_{0}(x)=K(x, 0)$ and $K_{1}(x)=K(x, 1)$ for every $x \in X$. But, the assumption aabout $\xi_{c}$ gives that the maps appearing in (39) and (40) are homotopic. Hence,

$$
f^{c} \circ p_{b}^{f(c)} \circ p^{b} \cong q_{d}^{c} \circ f^{d} \circ p_{b}^{f(d)} \circ p^{b},
$$

where $b>f(c), f(d)$. However, the system $|\mathscr{X}|$ satisfies the condition (E2) from the reference $[7$, p. 48$]$, so that an $a>b$ for which (35) holds surely exists.

Now we can define that $\theta$ acts on morphisms of the category $\mathscr{H} M$ (i.e., on homotopy classes of multi-nets) by the rule $\theta([\varphi])=[\boldsymbol{f}]$, where $[\boldsymbol{f}]$ denotes the equivalence class of $\boldsymbol{f}$ with respert to the equivalence relation $\sim$ (see $[7$, p. 6]).

Claim 5. The function $\theta$ is well-defined i.e., it does not depend on the choices of $\varphi, \lambda$, and $f^{c}$ in our description of $\boldsymbol{f}$.

Proof of Claim 5. Suppose that $\psi=\left\{G_{c}\right\}: X \rightarrow Y$ is multi-net homotopic to $\varphi$ and let the morphism $\boldsymbol{g}=\left(g,\left\{g^{c} \mid c \in C\right\}\right)$ of inverse systems $|\mathscr{X}|$ and $|g|$ be constructed from $\psi$ by the above procedure using in it $\mu$ instead of $\lambda$. We must show that $\boldsymbol{f}$ and $\boldsymbol{g}$ are equivalent, i. e., that for every $c \in C$ there is an $a>f(c), g(c)$ with

$$
f^{c} \circ p_{a}^{f(c)} \cong g^{c} \circ p_{a}^{g(c)}
$$

Let a $c \in C$ be given. Since $\varphi$ and $\psi$ are homotopic multi-nets, there is an 
index $s_{c} \in \tilde{Y}$ such that

$$
F_{t} \stackrel{\pi_{c}}{\cong} G_{u} \quad \text { for all } t, u>s_{c} .
$$

Since the functions $\lambda$ and $\mu$ are increasing and cofinal, there is a $d>c$ such that $\lambda(d), \mu(d)>s_{c}$. From (43), we get

$$
F_{\lambda(d)} \stackrel{\pi_{c}}{\cong} G_{\mu(d)}
$$

Let $H: X \times I \rightarrow Y$ be a $\pi_{c}$-small multi-valued function with $F_{\lambda(d)}(x) \subset H(x, 0)$ and $G_{\mu(d)}(x) \subset H(x, 1)$ for every $x \in X$. Hence, $q^{c} \circ H$ is an $\eta_{c}$-small multi-valued function and

$$
q^{c} \circ F_{\lambda(d)}(x) \subset q^{c} \circ H(x, 0) \text { and } q^{c} \circ G_{\mu(d)}(x) \subset q^{c} \circ H(x, 1)
$$

for every $x \in X$. Just as in the proof of Claim 4 there is a single-valued continuous function $N: X \times I \rightarrow Y_{c}$ with

$$
N \stackrel{\xi_{c}}{\cong} q^{c} \circ H
$$

On the other hand, since $\lambda(d)>\lambda(c)>l_{c}$ the functions $F_{\lambda(c)}$ and $F_{\lambda(d)}$ are joined by a $\pi_{c}$-small homotopy $L: X \times I \rightarrow Y$. It follows that $q^{c} \circ L$ is an $\eta_{c}$-small homotopy which satisfies

$$
q^{c} \circ F_{\lambda(c)}(x) \subset q^{c} \circ L(x, 0) \text { and } q^{c} \circ F_{\lambda(d)}(x) \subset q^{c} \circ L(x, 1)
$$

for every $x \in X$. Pick a single-valued continuous function $M: X \times I \rightarrow Y_{c}$ with

$$
M \stackrel{\xi_{c}}{=} q^{c} \circ L
$$

Similarly, there is a single-valued continuous function $P: X \times I \rightarrow Y_{c}$ together with a $\pi_{c}$-small homotopy $R: X \times I \rightarrow Y$ such that

$$
q^{c} \circ G_{\mu(c)}(x) \subset q^{c} \circ R(x, 0) \text { and } q^{c} \circ F_{\mu(c)}(x) \subset q^{c} \circ R(x, 1)
$$

for every $x \in X$, and

$$
P \stackrel{\xi_{c}}{=} q^{c} \cdot R
$$

In analogy with (35), we also have

$$
g^{c} \circ p^{g(c)} \stackrel{s t\left(\xi_{c}\right)}{=} q^{c} \circ G_{\mu(c)} .
$$

Let $M_{0}, M_{1}, N_{0}, N_{1}, P_{0}$ and $P_{1}$ be maps defined from maps $M, N$ and $P$ as we defined $K_{0}$ and $K_{1}$ from $K$ in the proof of Claim 4.

The relations (34) and (48) imply that $f^{c} \circ p^{f(c)}$ and $M_{0}$ are $s t^{2}\left(\xi_{c}\right)$-close maps 
into $Y_{c}$. It follows that they are homotopic. Similarly, the maps $g^{c} \circ p^{g(c)}$ and $P_{1}$ are homotopic.

The maps $M_{1}$ and $N_{0}$ are also homotopic because from relations (45)-(48) we see that both are $\xi_{c}$-close to the function $q^{c} \circ F_{\lambda(d)}$. The maps $N_{1}$ and $P_{0}$ are homotopic because of a similar reason.

We conclude from the last two paragraphs that maps $f^{c} \circ g^{f(c)}$ and $g^{c} \circ p^{g(c)}$ are homotopic. Just as in the proof of Claim 4, with the help of the condition (E2), we can conclude that there exists an $a>f(c), g(c)$ so that (42) holds.

Claim 6.

(1) Let $\iota=\left\{\left(i d_{X}\right)_{z \in \tilde{X}}\right.$ be the identity multi-net on a space $X$. Then the morphism $i:|\mathfrak{X}| \rightarrow|\mathfrak{X}|$ associated to c by our description of $\theta$ is the identity morphısm $\left(i d_{A},\left\{\left(i d_{X}\right)_{a} \mid a \in A\right\}\right)$.

(2) Let $\varphi=\left\{F_{i}\left\{: X \rightarrow Y\right.\right.$ and $\phi=\left\{G_{u}\right\}: Y \rightarrow Z$ be multi-nets. Then

$$
\theta([\psi] \circ[\varphi])=\theta([\psi]) \circ \theta([\varphi]) \text {. }
$$

(3) $\theta$ is a functor and the relation $S=\theta \circ J$ holds.

Proof of Claim 6 (2). Let $\eta=\left\{H_{u}\right\}: X \rightarrow Z$, where $H_{u}=G_{g(u)} \circ F_{f(g *(u))}$ for every $u \in \tilde{Z}$. Let $\boldsymbol{f}=\left(f,\left\{f^{c}\right\}_{c \in C}\right), \boldsymbol{g}=\left(g,\left\{g^{m}\right\}_{m \in M}\right)$, and $\boldsymbol{h}=\left(h,\left\{h^{m}\right\}_{m \in M}\right)$ be obtained from $\varphi, \phi$ and $\eta$ by the above procedure. We must show that $\boldsymbol{h}$ and $\boldsymbol{g} \circ \boldsymbol{f}$ are homotopic. Since $\boldsymbol{g} \circ \boldsymbol{f}=\left(f \circ g,\left\{g^{m} \circ \boldsymbol{f}^{\boldsymbol{g}(m)}\right\}\right)$, this amounts to show that for every $m \in M$ there is an $a>t, x$ such that

$$
h^{m} \circ p_{a}^{x} \cong g^{m} \circ f^{v} \circ p_{a}^{t},
$$

where $t=f \circ g(m), x=h(m)$ and $v=g(m)$.

Once again, our method is to show that

$$
h^{m} \circ p^{x} \cong g^{m} \circ f^{v} \circ p^{t},
$$

and then use the condition (E2) to get the required index.

In order to establish (53), we shall argue that there are large enough indices $b \in C$ and $n \in M$ such that

$$
\begin{gathered}
h^{m} \circ p^{x} \stackrel{s t\left(\nu_{m}\right)}{=} r^{m} \circ H_{y}, \\
r^{m} \circ H_{y} \stackrel{\mu_{m}}{\cong} r^{m} \circ G_{z} \circ F_{c}, \\
r^{m} \circ G_{z} \circ F_{c} \stackrel{\mu_{m}}{\cong} r^{m} \circ G_{n} \circ F_{c}, \\
r^{m} \circ G_{n} \circ F_{c} \stackrel{\mu_{m}}{\cong} r^{m} \circ G_{w} \circ F_{c},
\end{gathered}
$$




$$
\begin{gathered}
r^{m} \circ G_{w} \circ F_{c} \stackrel{s t^{2}\left(\nu_{m}\right)}{=} g^{m} \circ q^{v} \circ F_{c}, \\
g^{m} \circ q^{v} \circ F_{c} \stackrel{\mu_{m}}{\cong} g^{m} \circ q^{v} \circ F_{u},
\end{gathered}
$$

and

$$
g^{m} \circ q^{v} \circ F_{u} \stackrel{s t}{\stackrel{\left(\mu_{m}\right)}{=}} g^{m} \circ f^{v} \circ p^{t},
$$

where $y=\gamma(m), z=g(\gamma(m)), w=\kappa(m), u=\lambda(g(m)), \mu_{m}$ is analogous to $\eta_{c}$ and $\gamma, \kappa$ and $\lambda$ are functions used in constructing $\boldsymbol{h}, \boldsymbol{g}$ and $\boldsymbol{f}$, respectively.

Suppose for a moment that the relations (54)-(57) hold. From (55)-(57) it follows that there is a $s t^{2}\left(\mu_{m}\right)$-small multi-valued function $K: X \times I \rightarrow Z$ such that

$$
r^{m} \circ H_{y}(x) \subset K(x, 0) \text { and } r^{m} \circ G_{w} \circ F_{c}(x) \subset K(x, 1)
$$

for every $x \in X$. Similarly, from (59), it follows that there is a $\mu_{m}$-small multivalued function $L: X \times I \rightarrow Z$ with

$$
g^{m} \circ q^{v} \circ F_{c}(x) \subset L(x, 0) \text { and } g^{m} \circ q^{v} \circ F_{u}(x) \subset L(x, 1)
$$

for every $x \in X$. Let $B$ and $D$ be single-valued continuous functions such that

$$
B \stackrel{\nu_{m}}{=} K \quad \text { and } \quad D \stackrel{\nu_{m}}{=} L
$$

From [54), (61)] and (63), we get that maps $h^{m} \circ p^{x}$ and $B_{0}$ are $s t^{3}\left(\nu_{m}\right)$-close. Hence,

$$
h^{m} \circ p^{x} \cong B_{0} .
$$

Similarly, from (58) and (61)-(63), it follows that the two maps $B_{1}$ and $D_{0}$ are $s t^{3}\left(\nu_{m}\right)$-close. Hence,

$$
B_{1} \cong D_{0} .
$$

Finally, from (60), (62) and (63), we obtain that maps $D_{1}$ and $g^{m} \circ f^{v} \circ p^{t}$ are $s t^{3}\left(\nu_{m}\right)$-close. Hence,

$$
D_{1} \cong g^{m} \circ f^{v} \circ p^{t}
$$

The relations (64)-(66) together imply the relation (53). Thus it remains to explain why (54)-(60) hold. We shall describe what choice of $c$ and $n$ make each of these relations true and leave to the reader to put together all choices to pick them so that all are true simultaneously.

Add (54). This follows from the way in which $\boldsymbol{h}$ was constructed (it corresponds to the relation (34)).

Add (55). Observe that $H_{y}=G_{z} \circ F_{s}$, where $s=f\left(g^{*}(y)\right)$. Since $G_{z}$ is a 
$(\tilde{g}(y, z, z), \tilde{y})$-map (and therefore also a $(g *(y), \tilde{y})$-map because $g^{*}(y)$ refines $\tilde{g}(y, z, z))$ and $\tilde{y}$ refines $\rho_{m}=\left(r^{m}\right)^{-1}\left(\mu^{m}\right)$, it suffices to take $c>s$ because then $F_{s}$ and $F_{c}$ are joined by a $g^{*}(y)$-small homotopy $Q: X \times I \rightarrow Y$ so that $r^{m} \circ G_{z} \circ Q$ is a $\mu_{m}$-small homotopy joining $r^{m} \circ H_{y}$ and $r^{m} \circ G_{z} \circ F_{c}$.

Add (56), Let $n>z$. Then $G_{z}$ and $G_{n}$ are joined by a $(g(y, z, n), \tilde{y})$-map $R: Y \times I \rightarrow Z$. Hence, if $c>g^{*}(n)$, then $r^{m} \circ R \circ\left(F_{c} \times i d_{I}\right)$ is a $\mu_{m}$-small homotopy joining $r^{m} \circ G_{z} \circ F_{c}$ and $r^{m} \circ G_{n} \circ F_{c}$.

Add (57). Let $n>w$. Then $G_{n}$ and $G_{w}$ are joined by a $\rho_{m}$-small homotopy $T: Y \times I \rightarrow Z$. Let $\omega$ be a normal cover of $Y \times I$ such that $T$ is an $\left(\omega, \rho_{m}\right)$-map and let $\zeta$ be a normal cover of $Y$ obtained from $\omega$ by application of $[4$, p. 358]. Let $c>f(\{\zeta\})$. Then $F_{c}$ is a $\zeta$-small multi-valued function so that $r^{m} \circ T_{\circ}\left(F_{c} \times i d_{I}\right)$ is a $\mu_{m}$-small homotopy joining $r^{m} \circ G_{n} \circ F_{c}$ and $r^{m} \circ G_{w} \circ F_{c}$.

Add (58), First we observe that

$$
r^{m} \circ G_{w} \stackrel{s t\left(\nu_{m}\right)}{=} g^{m} \circ q^{v} .
$$

The relation (67) is just the version of the relation (34) for $\phi$. Choose a normal cover $\pi$ of $Y$ such that $G_{w}$ is a $\left(\pi, \rho_{m}\right)$-map. Let $c>f(\{\pi\})$. Then $F_{c}$ is $\pi$ small and the composition $r^{m} \circ G_{w} \circ F_{c}$ is $\mu_{m}$-small. Let $\beta=\left(g^{m} \circ q^{v}\right)^{-1}\left(\mu_{m}\right)$. Let $c>f(\{\beta\})$. Then $F_{c}$ is $\beta$-small and the composition $g^{m} \circ q^{v} \circ F_{c}$ is also $\mu_{m}$-small. With this information on the size of both sides appearing in (58), from (67), we can get (58).

Add (59), We can assume that $\xi_{v}>\left(g^{m}\right)^{-1}\left(\mu_{m}\right)$ for every $m \in M$. It might be necessary to pass from a given set of $\xi_{c}$ 's to the new ones by an inductive argument on number of predecessors in order to accomplish this. Let $c>u$. Then $F_{c}$ and $F_{u}$ are joined by a $\pi_{v}$-small homotopy $U: X \times I \rightarrow Y$ and $g^{m} \circ q^{v_{\circ}} U$ is a $\mu_{m}$-small homotopy between $g^{m} \circ q^{v} \circ F_{c}$ and $g^{m} \circ q^{v} \circ F_{u}$.

Add (60). The relation (34) for $c=v$ reads

$$
q^{v} \circ F_{u} \stackrel{s t\left(\xi_{v}\right)}{=} f^{v} \circ p^{t}
$$

Since $\xi_{v}>\left(g^{m}\right)^{-1}\left(\mu_{m}\right)$, we get from this the relation (60).

ProOF OF ClAIM 6 (3). That $\theta$ is a functor follows fram the previous discussion. It remains to see that $S=\theta \circ J$. Let $f: X \rightarrow Y$ be a map, i.e., a morphism of the category $I o p$. For each $c \in C$, there is a $\mu_{c} \in \xi_{c}^{* 2}$ such that $q$ is also a commutative uniform approximate resolution of $Y$ into the approximate inverse system $y^{\prime}=\left\{Y_{c}, \mu_{c}, q_{d}^{c}, C\right\}$. By Theorem (6.3) in [8], there is an approximate map $\boldsymbol{f}: \mathfrak{X} \rightarrow \mathfrak{y}^{\prime}$ such that $(\boldsymbol{p}, \boldsymbol{q}, \boldsymbol{f})$ is an approximate resolution of $f$. By Lemma (5.6) in [8], we get 


$$
f^{c} \circ p^{f(c)} \stackrel{\xi_{c}}{=} q^{c} \circ f .
$$

Let $\varphi_{f}=\left\{F_{i}\right\}: X \rightarrow Y$ be a multi-net, where $F_{i}=f$ for every $i \in \tilde{Y}$. Then $\left[\varphi_{f}\right]$ $=J(f)$. In applying the procedure from the description of $\theta$ to the multi-net $\varphi_{f}$ we can take for $\lambda$ a constant function and the above morphism $\boldsymbol{f}$. The relation (68) implies that $S=\theta \circ J$. Indeed, the induced morphisms satisfy

$$
|\boldsymbol{f}| \cdot|\boldsymbol{p}|=|\boldsymbol{q}| \circ f
$$

Since there is a unique morphism which satisfies (69), namely the morphism $S(f)$, we get $S(f)=\theta(J(f))$.

\section{Inverse of $\theta$}

We shall now prove that on spaces which admit ANR-resolutions with the onto projections (that we call O-spaces) the functor $\theta$ is a category isomorphism.

Definition 7. A space $X$ is called an O-space provided there is an ANRresolution $\boldsymbol{p}=\left\{p^{a}\right\}: X \rightarrow\left\{X_{a}, p_{b}^{a}, A\right\}$ in the sense of Mardešic [7], where each projection $p^{a}$ is an onto map.

At present we do not know what is the real extend of O-spaces. From results in [11], it follows that inverse limits of inverse systems of compact Hausdorff spaces with onto bonding maps are O-spaces. In particular, all compact metric spaces are O-spaces. One can easily check that the examples of non-degenerate regular spaces with the property that every real valued map on them is constant [5, p. 160] provide examples of spaces that are not O-spaces.

THEOREM 4. Let $X$ be a topological space and let $Y$ be an $O$-space. Then the function $\theta: \mathscr{H} M(X, Y) \rightarrow \mathcal{S h}(X, Y)$ is a bijection.

In order to prove Theorem 4, we shall construct the function $\zeta: \operatorname{Sh}(X, Y)$ $\rightarrow \mathscr{H} M(X, Y)$ which will be the inverse for the function $\theta$. The description of $\zeta$ and the verification of its properties is given below in Claims 7-10.

\section{Construction of the function $\zeta$}

Let $\boldsymbol{f}=\left(f,\left\{f^{c}\right\}_{c \in C}\right)$ be a morphism between inverse systems $|\mathfrak{X}|$ and $|\mathscr{y}|$. Let $s \in \tilde{Y}$. Recall that $s$ is a finite set of normal covers of $Y$ with the unique maximal element $\tilde{s} \in \hat{Y}$. By the condition (B1) for the approximate resolution 
$\boldsymbol{q}[8]$, there is an index $c(s) \in C$ such that

$$
\left(q^{c}\right)^{-1}\left(\boldsymbol{\xi}_{c}\right) \quad \text { refines } \tilde{s} \text { for every } c>c(s) \text {. }
$$

Let $\gamma: \tilde{Y} \rightarrow C$ be an increasing function with $\gamma(s)>c(s)$ for every $s \in \tilde{Y}$. Let $\varphi=\left\{F_{s}\right\}_{s \in \tilde{Y}}$, where $F_{s}=\left(q^{\gamma(s)}\right)^{-1} \circ f^{\gamma(s)} \circ p^{f(\gamma(s))}$.

Claim 7. The family $\varphi$ is a multi-net from $X$ into $Y$.

Proof of Claim 7. Let a $\sigma \in \hat{Y}$ be given. We must show that there is a $c \in \tilde{Y}$ such that

$$
F_{t} \stackrel{\sigma}{\cong} F_{s} \quad \text { for every } t>s .
$$

Let $s=\{\sigma\} \in \hat{Y}$. Let $t>s$. Put $m=\gamma(t), n=\gamma(s), v=f(m)$ and $w=f(n)$. Since $m>n$ and $\boldsymbol{f}$ is a morphism of inverse systems, there is an $a>v, w$ and a map $K: X_{a} \times I \rightarrow Y_{n}$ with

$$
K(x, 0)=q_{m}^{n} \circ f^{m} \circ p_{a}^{v}(x) \text { and } K(x, 1)=f^{m} \circ p_{a}^{w}(x)
$$

for every $x \in X_{a}$. Let $L=\left(q^{n}\right)^{-1} \circ K \circ\left(p^{a} \times i d_{I}\right)$. Then $L: X \times I \rightarrow Y$ is a $\sigma$-small homotopy. Moreover, for every $x \in X$, from (72), we get

$$
L(x, 0)=\left(q^{n}\right)^{-1} \circ q_{m}^{n} \circ f^{m} \circ p_{a}^{v} \circ p^{a}(x) \supset\left(q^{m}\right)^{-1} \circ f^{m} \circ p^{v}(x)=F_{t}(x),
$$

and

$$
L(x, 1)=\left(q^{n}\right)^{-1} \circ f^{n} \circ p_{a}^{w} \circ p^{a}(x)=\left(q^{n}\right)^{-1} \circ f^{n} \circ p^{w}(x)=F_{s}(x) .
$$

Hence, $L$ is a $\sigma$-small homotopy between $F_{t}$ and $F_{s}$.

Now we can define the function $\zeta$ by the rule $\zeta([f])=[\varphi]$.

ClaIM 8. The function $\zeta$ is well-defined, i.e., the value $\zeta([\boldsymbol{f}])$ does not depend on the choice of the representive $\boldsymbol{f}$ of the equivalence class $[\boldsymbol{f}]$ and on the choice of the function $\gamma$ in our description of $\varphi$.

Proof of Claim 8. Let $\boldsymbol{g}=\left(g,\left\{g^{c}\right\}_{c \in C}\right) \in[\boldsymbol{f}]$. Let $\phi=\left\{G_{s}\right\}_{s \in \widetilde{Y}}$ be constructed from $g$ by the above procedure using the increasing function $\mu: \tilde{Y} \rightarrow C$. We must show that $\varphi$ and $\psi$ are homotopic, i.e., that for every $\sigma \in \hat{Y}$ there is an $s \in \tilde{Y}$ such that

$$
F_{t} \stackrel{\sigma}{\cong} G_{t} \quad \text { for every } t>s \text {. }
$$

Let a $\sigma \in \hat{Y}$ be given. Let $\tau \in \sigma^{* 2}$. Put $s=\{\tau\} \in \tilde{Y}$. Pick an increasing function $\delta: \tilde{Y} \rightarrow C$ such that $\delta(t)>\gamma(t), \mu(t)$ for every $t \in \tilde{Y}$. 
Let $t>s$. Let $m=\gamma(t), n=\mu(t), k=\delta(t), u=f(m), v=f(k), y=g(n)$ and $z=$ $g(k)$. Since $k>m$, there is an $a>u, v$ with $f^{m} \circ p_{a}^{u} \cong q_{k}^{m} \circ f^{k} \circ p_{a}^{v}$. As in the proof of Claim 7 , we can conclude from here that

$$
F_{t} \stackrel{\tau}{\cong} K
$$

where $K=\left(q^{k}\right)^{-1} \circ f^{k} \circ p^{v}$. Similarly, we obtain

$$
L \stackrel{\tau}{\cong} G_{t}
$$

where $L=\left(q^{k}\right)^{-1} \circ g^{k} \circ p^{z}$. Since $\boldsymbol{f}$ and $\boldsymbol{g}$ are equivalent, there is a $b>v, z$ and a homotopy $H: X_{b} \times I \rightarrow Y_{k}$ with $H(x, 0)=f^{k} \circ p_{b}^{p}(x)$ and $H(x, 1)=g^{k} \circ p_{b}^{z}(x)$ for every $x \in X_{a}$. It follows that the composition $\left(q^{k}\right)^{-1} \circ H_{\circ}\left(p^{b} \times i d_{I}\right)$ is a $\tau$-small homotopy joining $K$ and $L$. This together with (77) and (76) implies (75).

Claim 9. For every morphism $\boldsymbol{f}=\left(f,\left\{f^{c}\right\}_{c \in C}\right):|\mathfrak{X}| \rightarrow \mid$ ag $\mid$ we have $|\boldsymbol{f}|=$ $\theta \circ \zeta([f])$.

Proof of Claim 9. For every $s \in \tilde{Y}$ choose an index $c(s) \in C$ such that (70) holds. Let $\gamma: \tilde{Y} \rightarrow C$ be an increasing function with $\gamma(s)>c(s)$ for every $s \in \tilde{Y}$. Let $\delta: C \rightarrow \tilde{Y}$ be a function such that $\delta(c) \in \gamma^{-1}(c)$ whenever $\gamma^{-1}(c) \neq \emptyset$. Let $\varphi=$ $\left\{F_{s}\right\}_{s \in \tilde{Y}}$, where $F_{s}=\left(q^{\gamma(s)}\right)^{-1} \circ f^{\gamma(s)} \circ p^{f(\gamma(s))}$. With respect to $\varphi$ we now choose $\eta_{c}$, $\pi_{c}$ and $l_{c}$ as we did in the description of the function $\theta$. Hence, we can assume that $\left(q^{r(m)}\right)^{-1} \circ f^{r(m)} \circ p^{f(r(m))} \stackrel{\pi_{c}}{\cong}\left(q^{\gamma(n)}\right)^{-1} \circ f^{r(n)} \circ p^{f(r(n))}$ whenever $m, n>l_{c}$.

Next, we shall select a cofinal increasing function $\lambda: C \rightarrow \tilde{Y}$ such that $\lambda(c)>$ $l_{c},\left\{\pi_{c}\right\}, \delta(c)$ for every $c \in C$. Let $u=\lambda(c), v=\gamma(u)$ and $w=f(v)$. Then $v>c$ and

$$
q^{c} \circ F_{u}=q^{c} \circ\left(q^{v}\right)^{-1} \circ f^{v} \circ p^{w}=q_{v}^{c} \circ f^{v} \circ p^{w} .
$$

Hence, in the next step, (i.e., the selection of the index " $f(c)$ " and the singlevalued continuous function " $f^{c}$ ") we can take some $z=g(c)$ with $z>w$ and the map $g^{c}=q_{v}^{c} \circ f^{v} \circ p_{z}^{w}$. It remains to check that the morphisms $\boldsymbol{f}$ and $\boldsymbol{g}=\left(g,\left\{g^{c}\right\}_{c \in C}\right)$ are equivalent. In other words, that for every $c \in C$ we can find an $a>f(c)$, $g(c)$ with

$$
f^{c} \circ p_{a}^{f(c)} \cong g^{c} \circ p_{a}^{f(c)}=q_{v}^{c} \circ f^{v} \circ p_{z}^{w} \circ p_{a}^{z} .
$$

But, this follows from the fact that $v>c$ and $\boldsymbol{f}$ is a morphism of inverse systems.

DEFINITION 8. Let $\sigma$ be a normal cover of a space $Y$. Two multi-valued functions $F, G: X \rightarrow Y$ are $\sigma$-hooked provided for every $x \in X$ there is an $S_{x} \in \sigma$ such that $S_{x}$ has non-empty intersection with both $F(x)$ and $G(x)$. 
Observe that $\sigma$-close multi-valued functions are $\sigma$-hooked.

LeMma 4. Let $F, G: X \rightarrow Y$ be multi-valued functions and let $\sigma$ be a normal cover of $Y$. If $F$ and $G$ are $\sigma$-small and $\sigma$-hooked, then $F \stackrel{\text { st }(\sigma)}{\cong} G$.

Proof of Lemma 4 . Since $F$ and $G$ are $\sigma$-small, there is a normal cover $\eta$ of $X$ such that for every $E \in \eta$ there are $S_{E}, T_{E} \in \sigma$ with $F(E) \subset S_{E}$ and $G(E) \subset T_{E}$. Define a function $H: X \times I \rightarrow Y$ by the rule $H(x, t)=F(x) \cup G(x)$ for every $x \in X$ and every $t \in I$. Let $\xi=\{E \times I \mid E \in \eta\}$. Clearly, $\xi$ is a normal cover of $X \times I$. We shall check that $H$ is a $(\xi, s t(\delta))$-map. This would imply that $H$ is a $\operatorname{st}(\sigma)$-small homotopy joining $F$ and $G$.

Then $H(K)=F(E) \cup G(E)=S_{E} \cup T_{E}$, for a member $K=E \times I$ of $\xi$ and $E \in \eta$. But, since $F$ and $G$ are $\sigma$-hooked, for every $x \in E$ there is an $R_{x} \in \sigma$ with $R_{x} \cap F(x) \neq \emptyset$ and $R_{x} \cap G(x) \neq \emptyset$. Hence, $H(K) \subset \operatorname{st}\left(R_{x}, \sigma\right)$.

ClaIM 10. For every multi-net $\varphi=\left\{F_{s}\right\}_{s \in \widetilde{Y}}: X \rightarrow Y$ we have $\zeta \circ \theta([\varphi])=[\varphi]$.

Proof of Claim 10. We first perform steps from the description of the functor $\theta$ to get $C_{c}, \pi_{c}, l_{c}, \lambda, f$, and the maps $f^{c}$. Then we perform steps from the description of $\zeta$ to get indices $c(s)$, the function $\gamma$, and a multi-net $\psi=$ $\left\{G_{s}\right\}_{s \in \widetilde{Y}}$, where $G_{s}$ is the composition $\left(q^{\gamma(s)}\right)^{-1} \circ f^{\gamma(s)} \circ p^{f(\gamma(s))}$. We must show that multi-nets $\varphi$ and $\phi$ are homotopic, i.e., that for every $\sigma \in \hat{Y}$ there is an $s \in \tilde{Y}$ with

$$
F_{t} \stackrel{\sigma}{\cong} G_{t} \quad \text { for every } t>s .
$$

Let a $\sigma \in \hat{Y}$ be given. Let $\tau \in \sigma^{* 3}$. Since $\varphi$ is a multi-net there is an $s>\{\tau\}$ such that

$$
F_{r} \stackrel{\sigma}{\cong} F_{t} \quad \text { for all } r, t>s .
$$

Let $t>s$. We shall prove that there is a large enough index $c \in C$ with the property that

$$
\begin{gathered}
F_{t} \stackrel{\tau}{\cong} F_{u}, \\
F_{u} \stackrel{s t^{2}(\tau)}{\cong}\left(q^{c}\right)^{-1} \circ f^{c} \circ p^{v},
\end{gathered}
$$

and

$$
\left(q^{c}\right)^{-1} \circ f^{c} \circ p^{v} \stackrel{\tau}{\cong} G_{t},
$$

where $u=\lambda(c)$ and $v=f(c)$. The relations (80)-(82) and Lemma 3 imply (78).

Add (80). Since $\lambda$ is a cofinal function, there is a $c \in C$ so that $\lambda(c)>s$. 
Then (80) is a consequence of (79).

Add (81). Let $c>\gamma(\{\tau\})$. If follows that $\left(q^{c}\right)^{-1}\left(\boldsymbol{\xi}_{c}\right)$ refines $\tau$ so that $\left(q^{c}\right)^{-1}\left(s t\left(\xi_{c}\right)\right)$ refines $s t(\tau)$. Hence, from the relation (34), we get

$$
\left(q^{c}\right)^{-1} \circ q^{c} \circ F_{u} \stackrel{s t(\tau)}{=}\left(q^{c}\right)^{-1} \circ f^{c} \circ p^{v}
$$

But, the composition on the left side of (83) is a $\pi_{c}$-small multi-valued function. Since $\pi_{c}=\left(q^{c}\right)^{-1}\left(\eta_{c}\right)$ refines $\tau$, by Lemma 4 , from (83) we get (81).

Add (82). Let $w=\gamma(t)$ and $z=f(w)$. Let $c>w, \gamma(\{\tau\})$. Since $\left(f,\left\{f^{d}\right\}_{d \in C}\right)$ is a morphism of inverse systems, there is an $a>v, z$ and a homotopy $H: X_{a} \times$ $I \rightarrow Y_{c}$ with $H(x, 0)=q_{c}^{V} \circ f^{c} \circ p_{a}^{v}(x)$ and $H(x, 1)=f^{w} \circ p_{a}^{2}(x)$ for every $x \in X_{a}$. It follows that $\left(q^{c}\right)^{-1} \circ H \circ\left(p^{a} \times i d_{I}\right)$ is a $\tau$-small homotopy joining the left and the right side of (82).

REMARK. It is possible to use only multi-valued functions that are upper semi-continuous or to require that in addition images of points are compact. With these functions we shall get a similar result but the space $Y$ is further restricted to spaces that admit ANR-resolutions with closed and perfect projections, respectively.

\section{References}

[1] R.A. Aló and M.L. Shapiro, Normal Topological Spaces, Cambridge Univ. Press, Cambridge, 1972.

[2] K. Borsuk, Concerning homotopy properties of compacta, Fund. Math. 62 (1968), 223-245.

[3] K. Borsuk, Theory of Shape, Monografie Matem. 59, Polish Scientific Publishers, Warszawa, 1977.

[4] A. Dold, Lectures on Algebraic Topology, Springer-Verlag, Berlin, 1972.

[5] R. Engelking, General Topology, Monografie Matem. 60, Polish Scientific Publishers, Warszawa, 1977.

[6] S. T. Hu, Theory of Retracts, Wayne State University Press, Detroit, 1965.

[7] S. Mardešić and J. Segal, Shape Theory, North Holland, Amsterdam, 1982.

[8] S. Mardešić and T. Watanabe, Approximate resolutions of spaces and mappings, Glasnik Mat. 24 (1989), 583-633.

[9] J. Sanjurjo, Shape morphisms and small multivalued maps, Math. Japonica 35 (1990), 713-717.

[10] J. Sanjurjo, An intrinsic description of shape, Trans. Amer. Math. Soc. 329 (1992), 625-636.

[11] T. Watanabe, Approximate Shape, I, Tsukuba J. Math. 11 (1987), 17-59.

Kopernikova 7, 10020 Zagreb,

Croatia, Europe

E-mail : zcerin@x400.srce.hr 